\title{
THE IMPACT OF REMOTE UROLOGY OUTPATIENT CLINICS DURING THE COVID-19 PANDEMIC
}

Yehia Abdelmotagly ${ }^{1,3}$, Mohamed Noureldin ${ }^{1,2}$, Louise Paramore ${ }^{1}$, Govindaraj Rajkumar ${ }^{1}$, Timothy Nedas ${ }^{1}$, Naomi Neal ${ }^{1}$, Richard Hindley ${ }^{1}$, Amr Emara ${ }^{1,2}$

1. Hampshire Hospitals NHS Foundation Trust, United Kingdom

2. Urology Department, Ain Shams University, Cairo, Egypt

3. Urology Department, Misr University for Science and Technology, Giza, Egypt

\begin{abstract}
Introduction: The coronavirus (COVID-19) pandemic of 2020 had a major impact on NHS services. From the 23rd of March 2020, the Urology Department in Basingstoke initiated telephone-led consultation clinics instead of face-to-face outpatient appointments, in accordance with U.K. guidance.

Objectives: To evaluate patient experience and satisfaction following the introduction of remote (telephone) consultations during the COVID-19 pandemic.

Patients and methods: The first 200 remote patient appointments between the 30th of March 2020 and the 16th of April 2020 were sent a postal questionnaire (19 questions relating to their experience and level of satisfaction with the interaction). Telephone consultations were conducted by 6 consultants, 3 registrars, and 2 specialist nurses. The patients were not prewarned to expect a questionnaire after the remote appointment. The associated cost saving resulting from a switch from face-to-face appointments to remote telephone appointments was also calculated.

Results: 100 out of the 200 patients responded within 1 month (response rate $50 \%$ ). A total of $44 \%$ of the patients were new referrals, while $56 \%$ were follow-ups. Overall, the feedback was positive regarding the telephone consultation, with $88 \%$ rating the care received as excellent or very good. In addition, $90 \%$ would recommend a telephone consultation to family and friends. However, $35 \%$ would prefer in the future to have another telephone consultation rather than face-to-face consultation, with $46 \%$ preferring a face-to-face appointment in the future and $19 \%$ unsure. For new patients, the proportion wishing to have a face-to-face appointment, in the end, was unsurprisingly higher than it was for those undergoing a follow-up (39\% vs. $7 \%$ ). In these 2 weeks, the cost reduction to the NHS from shifting from face-to-face consultation to telephone consultation was estimated to be $£ 6500$.

Conclusions: Telephone urology clinics are a satisfactory alternative to face-to-face appointments for many of our patients now and beyond the COVID-19 pandemic. They are efficient, cost-effective, and feasible to undertake urological consultation and can be implemented successfully in selected patients. The feedback from this questionnaire would suggest that priority should be given to face-to-face appointments for new patients and for complex follow-up appointments. Telephone follow-up appointments, however, are a good approach for the majority of patients.
\end{abstract}

Keywords: COVID-19; SARS-CoV-2; Urology; Telehealth; NHS 


\section{INTRODUCTION}

The impact and unforeseeable challenges on the NHS during the COVID-19 pandemic necessitated a shift (from the 23rd of March 2020) from faceto-face into remote consultations using electronic communication tools. ${ }^{1}$

Face-to-face consultations have long been the heritage of urology consultations as they provide a safe and private environment for the patient. Psychologist Albert Mehrabian illustrated the importance of nonverbal aspects of communication in the research of salespersons, quoting $7 \%$ attributable to spoken words, $55 \%$ body language, and $38 \%$ voice and tone. ${ }^{2}$ The doctor-patient relationship is assembled around these aspects, which creates trust and compliance.

Telehealth refers to any health care activity performed by telecommunication. According to the Telehealth Resource Centre, a leading consortium of telehealth networks includes "a collection of means or methods for enhancing health care, public health, and health education delivery and support using telecommunications technologies."

Switching to remote (telephone) consultations was the main goal during the coronavirus disease 2019 (COVID-19) pandemic, according to the Public Health England guidance and British Association of Urological Surgeons (BAUS) recommendations. ${ }^{4}$ Implementing that was to comply with quarantine strategies and avoid patients from catching the infection, limit the exposure, reduce the spread and lower infection rates, providing protection for both the patient and the healthcare professionals while delivering optimal urology care. ${ }^{5}$

Understanding that clinical history is a critical determinant of diagnosis means that remote consultations should adequately help many patients. ${ }^{6}$ When patients were booked for a telemedicine clinic, they were given information in their appointment letter about the timing of the clinic that they will have the consultation at a certain time with a possibility of one hour on either side of the appointment, recommendation to take notes and to be within a quiet environment during the call.

In Hampshire Hospitals NHS Foundation Trust, as with many other Trusts in the U.K., we had already transitioned to electronic records and prescribing systems before the Covid-19 pandemic. Such digital pathway transformation has underpinned the ability to deliver telephone clinics within the NHS. ${ }^{7}$

This study aimed to assess the effectiveness of telephone clinics, including patient satisfaction, and identify any areas for improvement.

\section{PATIENTS AND METHODS}

We retrospectively extracted data at the beginning of the pandemic between 30th of March 2020 and 16th of April 2020 for a group of 200 patients. An anonymous patient questionnaire was sent by post, of whom 100 patients responded.

The questionnaire included 3 domains:

1. Referral type; the time between referral and appointment and the effect of this waiting time on the patient's condition.

2. Quality of the appointment itself; timing of the call, duration of the call, the caller's ability to explain and understand the patient's complaints and concerns.

3.Patient's assessment of appointment; satisfaction, recommendations to others, and the type of preferred follow-up appointment. See Appendix (1).

We calculated the cost of new and follow-up faceto-face and telephone appointments using Trust tariffs.

All the data from questionnaires returned within one month was used for analysis.

\section{STATISTICS}

All extracted data were analyzed using IPSS IBM Software version 17.

Consultations were conducted by 11 members of the urology team, including 6 consultants $(58 \%$ of consultations), 3 registrars (36\%), and 2 specialist nurses $(6 \%)$.

The overall questionnaire response rate was $50 \%$. From the 100 responses, 44 patients were new referrals ( 5 haematuria, 12 prostate cancer, 3 renal, 20 LUTS, 2 stone disease, and 2 testicular) while 56 were follow-up appointments

Despite the pandemic, patients were reviewed quickly, with $42 \%$ waiting less than 4 weeks between referral and appointment. Only $28 \%$ waited more 
than 3 months. Interestingly, $69 \%$ of them reported no symptoms to change during this waiting period. Also, the less the waiting time, the higher the overall satisfaction about the whole consultation. For example, $24 \%$ rated the consultation as excellent in those who waited less than 1 month, while only $12 \%$ gave that rate if they waited 3 months or more.

During the consultation itself, $51 \%$ of our appointments started at the scheduled time, $46 \%$ within an hour, and a few patients could not comment on the exact timing.

In the telephone appointment, as with a face-to-face appointment, the real challenge to the physician is extracting all the necessary information, understanding the patient's concerns, and discussing the management plan within the allocated time. Although 71\% of our consultations lasted 10 minutes or less, $88 \%$ of patients rated as excellent or good the discussing their condition. Also, 91\% explained that the caller managed to explain the reasons for any treatment or action either in a complete way $(78 \%)$ or to some extent (13\%). Only one patient reported that the caller did not listen carefully to what the patient had to say. $98 \%$ reported that they were as involved as they wanted in the decisions taken in their case during this telephone appointment ( $77 \%$ completely and $21 \%$ to some extent).

After the appointment, condition-related information leaflets were sent to some patients to further improve patients understanding of their diagnosis and management. $22 \%$ of our patients reported receiving written information after their appointments.

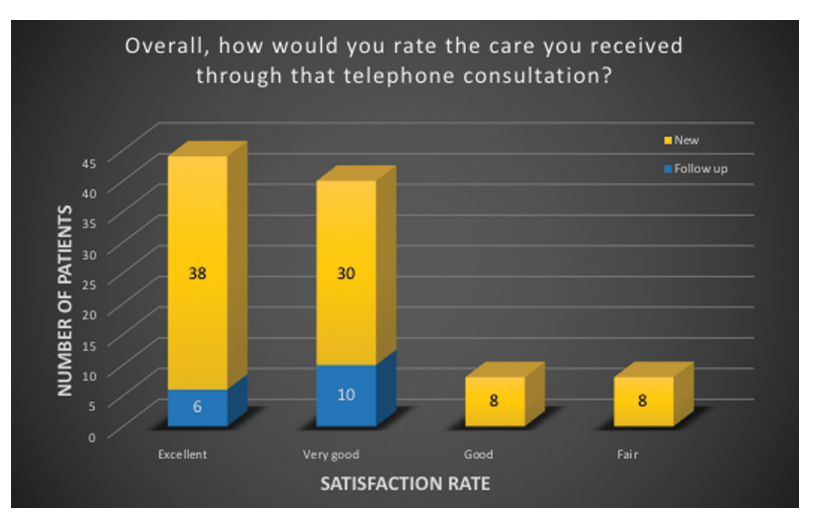

Graph 1. Satisfaction rate.

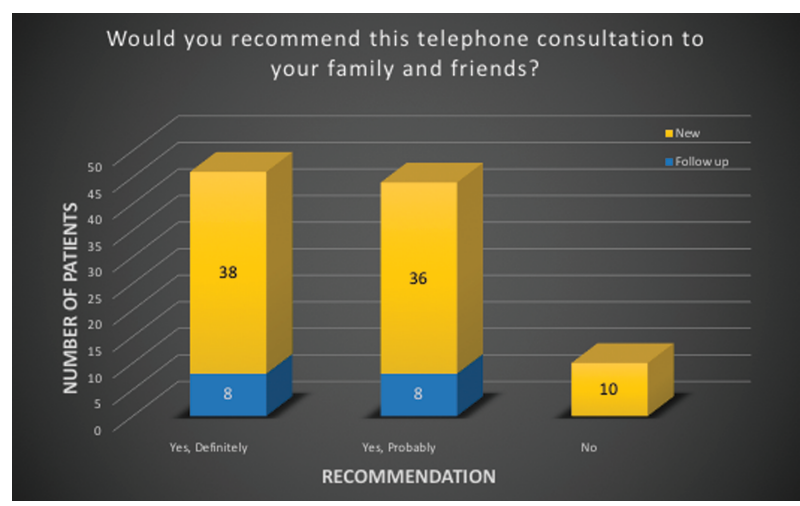

Graph 2. Recommendation rate.

Patient satisfaction regarding the service received was assessed through the questionnaire using multiple questions. $92 \%$ positively rated the service (44\% excellent, $40 \%$ very good and $8 \%$ good). No one reported the appointment as bad, only $8 \%$ experienced a fair service, and all were new referrals (Graph 1). Most of the patients (90\%) said they would recommend the telephone consultation to their families or friends, and the rest will not (Graph 2). However, almost half of our patients $(46 \%)$ asked to have their next face-toface appointment; these patients included 6 patients as a new referral on a 2-week pathway rule, 12 new routine referrals, and 28 were follow-ups. A total of $35 \%$ were happy with another telephone appointment, and $19 \%$ did not know their preference for follow-up appointments. (Graph 3).

Our Trust tariffs were used to estimate the potential cost-savings of telephone clinics. For example, a

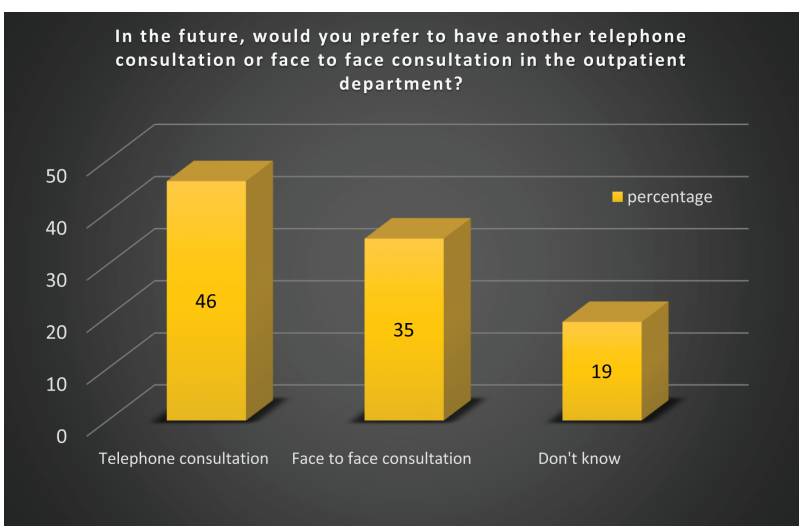

Graph 3. Preference rate.

J Endolum Endourol Vol 4(3):e17-e25; November 19, 2021.

This article is distributed under the terms of the Creative Commons Attribution-Non Commercial 4.0 International License. (C) Abdelmotagly, et al. 
new face-to-face appointment with a consultant will cost the Trust $£ 154$ while only $£ 105$ for a telephone appointment. The follow-up will cost $£ 76$ and $£ 51$, respectively. In non-consultant new appointments, these cost $£ 145$ as a face-to-face and $£ 48$ as a telephone. The follow-ups will be $£ 63$ and $£ 48$, respectively. This means that there was a net saving of $£ 6500$ to the Trust for these 100 appointments.

\section{DISCUSSION}

The COVID-19 pandemic has created a significant challenge for all healthcare systems. A delicate balancing act is required, which provides beds to the COVID-19 hospitalised patients and provides the necessary medical care for the acute and elective illnesses in the community. Cancer services, including urological malignancies, are usually under pressure and can delay providing a service in a timely manner for patients. ${ }^{8}$ Providing this remote telephone consultation for triaged patients saved substantial time in lieu rather than waiting to have a face-to-face consultation and avoided many cancellations. ${ }^{7}$ Subsequent services such as imaging, prescriptions, and blood tests that rely mainly on the results of the primary consultation could be arranged safely following that telephone appointment, minimising the number of attendances required in the hospital. ${ }^{7}$

Patient satisfaction with the shift from face-to-face to telephone consultation should be further assessed after the COVID-19 era to identify whether the satisfaction rates are consistent during normal times. ${ }^{9}$

Previous studies have explored the utilisation of telephone clinics, confirming improved ability to save time for other services with overall improved efficacy. ${ }^{7}$ Another study mirrored the cost-benefit identified in our research with the added benefits of reducing environmental impact and minimising travel hazards. ${ }^{10}$ Such wider benefits are important, particularly since the NHS is responsible for $5 \%$ of the U.K.'s road transport emission-related journeys, as undertaken by keyworkers, patients, and guests. ${ }^{11}$ There are growing numbers of hospital trusts which use remote consultations to lessen the amount of travel by patients and staff, reducing the cost, shortening distance travelled, thereby reducing missed work time, such as The Royal Cornhill Hospital in
Aberdeen. ${ }^{11}$ Future studies are likely to look at the impact on different communities, including those in more rural areas.

Although our study was a short-term outcome measuring the effectiveness of the telephone clinics over only a few weeks, it showed a significant cost reduction (£6500) to the Trust. Although not specifically looked at, we anticipate a cost-saving to the patient by not traveling to the hospital. Moreover, the carbon footprint is also reduced by avoiding these 100 patients making journeys to and from the hospital. If such figures are replicated across other departments within a Trust, the annual cost savings would be substantial.

Previously published studies show remote consultations are associated with a high level of patient satisfaction, $80-90 \% .^{7,12}$ In our study, $92 \%$ were satisfied with the service they received. Patients also appreciate the effort provided by healthcare workers to ensure safe boundaries and provide a consultation service away from the Covid inpatient population. Viers et al. ${ }^{13}$ surveyed all adult urology outpatients over six months. In addition, an online survey was performed with a total of 1378 (25\%) completing it; 63\% (868/1378) 'likely', 12\% (172/1378) 'neutral' and $25 \%(338 / 1378)$ 'unlikely' to accept remote consultations.

It must be recognised that some patients will still prefer to have face-to-face consultation ( $46 \%$ of our patients). The reasons behind this include the need for physical examination (e.g., inguinoscrotal and penile referrals) or a language/hearing barrier. Therefore, triaging patients and sending detailed instructions of what they could expect from remote consultation is critical in facilitating the success of this service. ${ }^{14}$ Interpreting services were employed in a minority of our telephone consultations with good success, but they require advance planning. This includes contacting the telephone interpreting service (e.g., Language line) in advance of the patient's appointment and providing a call back number for the doctor leading the consultation. Despite evidence suggesting the utility and effectiveness of remote consultations, telehealth modalities are sparse as it was forced during the pandemic shielding conditions and was not applicable to this volume of patients in our institute before the pandemic. ${ }^{15}$ 
Appropriately trained staff to deliver the required medical care through remote service is essential to maintain the standards for these telephone clinics. Although our study had a range of medical staff delivering these clinics with good patient satisfaction, none of the staff underwent any specific training in this type of clinic, and none of the telephone consultations included training of medical students or junior staff. This is clearly an area for further development to provide appropriate training for junior doctors and medical students. This could be addressed as we look to continue this service, particularly concerning teaching medical students and other health professionals. Other factors such as availability of I.T. support, patient systems software, and a confidential, secure system to keep patient data protected are indispensable elements in running this service. ${ }^{16}$

\section{CONCLUSION}

Telemedicine urology clinics are an acceptable and promising new platform for many of our patients. They are a safe, efficient, and feasible way of undertaking consultation and can be implemented successfully in a selected large sub-group of patients, without compromising patient safety; for example, for non-complex follow-ups patients, or for triaging new appointments and directing them to imaging or other investigations before face-to-face review where appropriate. Adequately planning the time given for each appointment and the spacing between appointments is essential for staff and patients. A patients' understanding of their condition should be explored as much as possible during the call. Written information leaflets could be sent by post after the consultation and should be encouraged if required, in addition to a copy of the G.P./patient letter.

Many urological conditions are suitable for telehealth, but more studies are needed. Nevertheless, it is likely that out of adversity, the COVID-19 pandemic will give a significant boost to the use of digital health services and strategies in the future.

More robust data on long-term efficacy, time consumption, reduction of resource usage and costeffectiveness are essential.

\section{DECLARATIONS OF INTEREST}

None.

\section{REFERENCES}

1. Hollander JE, Carr BG. Virtually perfect? Telemedicine for COVID-19. N Engl J Med. 2020.

2. Mehrabian A, Ferris SR. Inference of attitudes from nonverbal communication in two channels. J Consult Psychol. 1967.

3. Center for Connected Health Policy. What is Telehealth? Website. cchpca.org. 2018.

4. Public Health England. Guidance on social distancing for everyone in the U.K. Guid Soc distancing everyone U.K. 2020.

5. Chan MC, Yeo SEK, Chong YL, Lee YM. Stepping forward: Urologists' efforts during the COVID-19 outbreak in Singapore. Eur Urol. 2020.

6. Hampton JR, Harrison MJG, Mitchell JRA, Prichard JS, Seymour C. Relative contributions of history-taking, physical examination, and laboratory investigation to diagnosis and management of medical outpatients. $\mathrm{Br}$ Med J. 1975.

7. Connor MJ, Miah S, Edison MA, Brittain J, Smith MK, Hanna M, et al. Clinical, fiscal and environmental benefits of a specialist-led virtual ureteric colic clinic: a prospective study. BJU Int. 2019.

8. Russell B, Liedberg F, Khan MS, Nair R, Thurairaja R, Malde $\mathrm{S}$, et al. A Systematic review and meta-analysis of delay in radical cystectomy and the effect on survival in bladder cancer patients. Eur Urol Oncol. 2020.

9. Hanlon P, Chadwick F, Shah A, Wood R, Minton J, McCartney G, et al. COVID-19 - exploring the implications of long-term condition type and extent of multimorbidity on years of life lost: a modelling study. Wellcome Open Res. 2020.

10. Miah S, Dunford C, Edison M, Eldred-Evans D, Gan C, Shah TT, et al. A prospective clinical, cost and environmental analysis of a clinician-led virtual urology clinic. Annal Royal College Surg Engl. 2019.

11. Mayor S. NHS should bring in measures to reduce its carbon footprint, BMA says. BMJ (Clinical research ed.). 2008.

12. Vijayasundaram S, Karthikeyan P, Mehta SD. Proficiency of virtual follow-up amongst tinnitus patients who underwent intratympanic steroid therapy amidst COVID 19 pandemic. Am J Otolaryngol - Head Neck Med Surg. 2020. 
13. Viers BR, Pruthi S, Rivera ME, O’Neil DA, Gardner MR, Jenkins SM, et al. Are patients willing to engage in telemedicine for their care: A survey of preuse perceptions and acceptance of remote video visits in a urological patient population. Urology. 2015.

14. Connor MJ, Winkler M, Miah S. COVID-19 pandemic - is virtual urology clinic the answer to keeping the cancer pathway moving? BJU Internat. 2020.
15. Wallace P, Haines A, Harrison R, Barber J, Thompson S, Jacklin P, et al. Joint teleconsultations (virtual outreach) versus standard outpatient appointments for patients referred by their general practitioner for a specialist opinion: A randomised trial. Lancet. 2002.

16. Bokolo AJ. Exploring the adoption of telemedicine and virtual software for care of outpatients during and after COVID-19 pandemic. Irish J Med Sci. 2020. 


\section{(APPENDIX 1) \\ UROLOGY TELEPHONE CLINIC QUESTIONNAIRE}

\section{What is the survey about?}

This survey is about your most recent urology telephone appointment through Basingstoke and North Hampshire Hospital

\section{Who should complete this questionnaire?}

The questions should be answered by the person named on the front of the envelope. If that person needs help to complete the questionnaire, the answers should be given from his/her point of view - not from the point of view of the person who is helping.

\section{Completing the questionnaire}

Please tick clearly inside one box using a black or blue pen.

Don't worry if you make a mistake; simply cross out the mistake and put a tick in the correct box.

Please do not write your name or address anywhere on the questionnaire.

Taking part in this survey is voluntary. Your answers will be treated in confidence.

This questionnaire is about your most recent telephone consultation by the urology department

1. Have you ever had a telephone consultation by the urology department before for the same condition?

$\square$ Yes

$\square$ No

2. From the time you were first told you needed an appointment to the time you received the call; how long did you wait for your appointment?

$\square$ Up to 1 month

$\square 1$ month to 6 weeks

More than 6 weeks but no more than 3 months

More than 3 months but no more than 5 months

More than 5 months but no more than 12 months

More than 12 months but no more than 18

months
More than 18 months

I received the call without an appointment

Don't know / Can't remember

3. Did your symptoms or condition get worse while you were waiting for your appointment?

$\square$ Yes, definitely

$\square$ Yes, to some extent

No

Don’t know / Can’t remember

4. Before your appointment, did you know the reason for the appointment?

Yes, definitely

Yes, to some extent

No 
5. How long after the stated appointment time did the appointment start?

$\square$ Called on time, or early

Waited up to 5 minutes

Waited 6 - 15 minutes

Waited 16 - 30 minutes

Waited 31 - 60 minutes

Waited more than 1 hour but no more than 2 hours

Waited more than 2 hours

Don't know / Can't remember

6. Were you told how long you would have to wait?

$\square$ Yes, but the wait was shorter

Yes, and I had to wait about as long as I was told

Yes, but the wait was longer

No, I was not told

Don't know / Can't remember

7. Did you have enough time to discuss your health or medical problem with the doctor over the phone?

$\square$ Yes, definitely

$\square$ Yes, to some extent

No

8. Did the doctor seem aware of your medical history?

$\square \mathrm{He} / \mathrm{she}$ knew enough

$\square \mathrm{He} /$ she knew something but not enough

$\square \mathrm{He} / \mathrm{she}$ knew little or nothing

$\square$ Don't know / Can’t say

9. How long did the call last?

$\square \quad$ Up to 5 minutes

$\square \quad 6-10$ minutes

$\square$ 11- 20 minutes

$\square 21$ - 30 minutes

More than 30 minutes

Can't remember

10. Did the doctor explain the reasons for any treatment or action in a way that you could understand?

$\square$ Yes, completely

Yes, to some extent

No

I did not need an explanation

No treatment or action was needed
11. Did the doctor listen to what you had to say?

Yes, definitely

Yes, to some extent

No

12. Did you receive any information (e.g. leaflets, other types of media) after your appointment?

$\square$ Yes

$\square$ No

13. Did the staff calling you introduce themselves?

$\square$ Yes, they did

$\square$ No, they didn't

Don't know / Can't remember

14. Were you involved as much as you wanted to be in decisions about your care and treatment?

$\square$ Yes, definitely

Yes, to some extent

No

15. Did your appointment help you to feel that you could better manage your condition or illness?

Yes, definitely

Yes, to some extent

No

This was not necessary

16. Overall, how would you rate the care you received through that telephone consultation?

$\square$ Excellent

$\square$ Very good

$\square$ Good

$\square$ Fair

$\square$ Poor

Very poor

17. Would you recommend this telephone consultation to your family and friends?

$\square$ Yes, definitely

$\square$ Yes, probably

No 
18. In the future, would you prefer to have another telephone consultation or face to face consultation in the outpatient department?

$\square$ Telephone consultation

$\square$ Face to face appointment

$\square$ Don't Know

\section{ANY OTHER COMMENTS}

If there is anything else, you would like to tell us about your experiences with the urology telephone consultation please do so here.

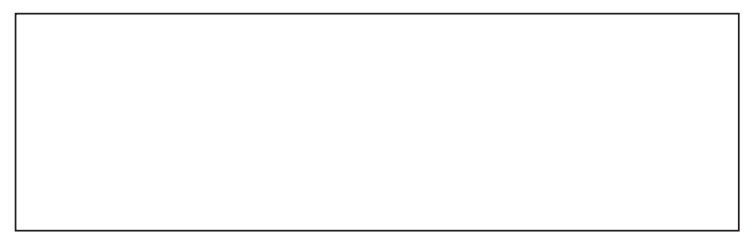

THANK YOU VERY MUCH FOR YOUR HELP

Please check that you answered all the questions that apply to you.

Please post this questionnaire back in the STAMPED envelope provided. 